\title{
TIME SERIES CATEGORIZATION OF DRIVING MANEUVERS USING ACCELERATION SIGNALS
}

\author{
Chris Schwarz \\ National Advanced Driving Simulator, University of Iowa, Iowa City, IA, USA \\ Email: chris-schwarz@uiowa.edu
}

\begin{abstract}
Summary: Two methods of time series analysis were applied to naturalistic driving data. The SAX method reduces the dimensionality of the data by discretizing and quantizing it into distinct symbols. The matrix profile method works on raw data and computes a Euclidian distance measure between subsequences of the time series. Both methods can be used to search for motifs and discords (anomalies) in the data. We discuss the applications of these methods to look for driving patterns and show an example of a left turn that was identified using both methods. After comparing the methods, the matrix profile was the preferred method.
\end{abstract}

\section{BACKGROUND}

Foundational to many applications of driver modeling and data mining of vehicle data is the ability to detect and categorize patterns that appear in the data. It has been shown that driving behavior and individual 'fingerprints' of driving can be identified with no more than smartphone sensors (Enev, Takakuwa, Koscher, \& Kohno, 2016). Many opportunities to identify driving patterns exist with naturalistic data that captures drivers' actual day-to-day actions (Jovanis, Aguero-Valverde, Wu, \& Shankar, 2011).

The modeling of driver impairment has been conducted using not only driver-based sensors (eye tracking), but also vehicle-based ones (steering, pedals). This approach has been used at the National Advanced Driving Simulator (NADS) by applying machine learning techniques to identify driving patterns unique to alcohol (Lee et al., 2010), drowsiness (Brown, Lee, Schwarz, Fiorentino, \& McDonald, 2014) and distraction (Schwarz, Brown, \& Gaspar, 2016).

\section{Unintended Acceleration}

Of particular interest in this work is the problem of sudden unintended acceleration (SUA). Many such events turn out to be caused by driver pedal errors. A good overview of the history of SUA is publicly available in a NHTSA report (Lococo, Staplin, Martell, \& Sifrit, 2012). While early investigations of pedal errors resulted in the mandating of shift-brake interlocks, the problem frustratingly persists today.

A vision analysis of driver foot gestures was used to predict pedal errors (Tran, Doshi, \& Trivedi, 2011), and this is significant because the data mining approaches discussed herein can be applied equally well to many kinds of data, including gestures. Video coding of driver feet was also applied in a naturalistic study (McGehee et al., 2014). Yu and Langari developed a detection and warning system for SUA by testing thresholds of acceleration and braking given the acceleration and speed of the vehicle (Yu \& Langari, 2015). 


\section{Time series data mining}

Time series data consist of signals that are (usually) sampled at regular time intervals, and include signals such as speed, position and acceleration for driving data. We are often interested in asking questions of such data including: are there repetitive patterns in the data (motif discovery), are there anomalous patterns in the data (discord discovery), are there patterns that are uniquely representative of the data (shapelet discovery), and can the data be separated naturally into different regimes (semantic segmentation)?

Symbolic Aggregate Approximation (SAX). The SAX technique is a symbolic representation of a time series that codes continuous data into quantized levels and then into discrete symbols, usually a subset of the alphabet (Lin, Keogh, Wei, \& Lonardi, 2007). This technique has the benefit of greatly compressing the original data into a smaller size at the expense of resolution. The resulting symbolic data can then be processed by natural language processing (NLP) techniques that can identify words and topics. A technique called randomized projections can be used to search for motifs in the data (Chiu, Keogh, \& Lonardi, 2003). The SAX method has been adapted and applied to driving data in recent years in efforts to apply NLP methods (A. McDonald et al., 2013; McLaurin et al., 2014).

The Matrix Profile. A more robust approach for mining time series data would not sacrifice its resolution by compressing it as SAX does. However, these types of algorithms have been plagued by the sheer computational burden of the problem. Running a search that could be used to identify motifs in big datasets could take months or more. Recent work on the problem has resulted in ultra-fast algorithms that can do just this (Mueen, Hamooni, \& Estrada, 2014; Rakthanmanon et al., 2013). The matrix profile is a computed data signal in which each sample represents the distance of a subsequence to its nearest neighbor. A subsequence length must be specified just like with SAX. Distance is computed by some metric, like Euclidian distance. An accompanying data signal is also generated, called the profile index. The values in the profile index give the index in the dataset of the nearest neighbor whose distance is recorded in the matrix profile.

\section{OBJECTIVES}

The purpose of this paper is to demonstrate the applications of the SAX and matrix profile methods to a naturalistic dataset and identify geo-spatial driving patterns in it. We were interested in identifying and classifying driving patterns that typically involve accelerations. These include maneuvers like turning, stopping at intersections, parking, and leaving parking spaces. It is in these situations where pedal errors are most likely to occur as drivers' feet move from one pedal to the other. If normal driving patterns are well understood, then detecting the onset of SUA becomes a matter of detecting anomalies in the driving pattern. This paper describes the process of finding motifs, or common patterns, in such data.

\section{METHODS}

The data used for this research were collected through a University of Iowa naturalistic driving study centered on understanding driver foot pedal behavior. Data were collected from September 
2013 through August 2014, where data collection systems were installed in owners' vehicles for a period of four weeks. An event recorder was developed for the instrumentation and recorded vehicle signals related to position, velocity and acceleration. A total of 34 participants completed the study. One third of participants were from the age group of 25-35 years and two thirds were over the age of 65 years. The project included video coding of the driver's feet, looking for pedal errors. In addition to video of the driver, forward view, and foot well, other collected data included GPS data that was recorded continuously (at 10hz frequency) while the vehicle is being operated, accelerometer data, and on-board diagnostics (OBD) data all collected at a $10 \mathrm{~Hz}$ sampling rate.

\section{Accelerometer Normalization}

When the longitudinal acceleration signal was checked against the filtered derivative of the speed in the naturalistic dataset, a scale difference was observed. The accelerometer signal was consistently smaller in magnitude than its estimate from speed. Then it was observed that a significant piece of the acceleration cues was present in the vertical acceleration signal. It is not known whether the accelerometer was installed at an angle in the car, or if it was mounted on a flexible surface that flexed when the car was under acceleration.

An attempt was made to normalize the longitudinal acceleration signal through a rotational transformation. A three-dimensional rotational transformation matrix was constructed by multiplying three constituent two-dimensional rotations in a standard Z-Y-X configuration. Let $A_{x}$ denote the roll rotation matrix, $A_{y}$ the pitch rotation matrix, and $A_{z}$ the yaw rotation matrix. Then the final transformation matrix, $A$, is given by $A_{z} A_{y} A_{x}$.
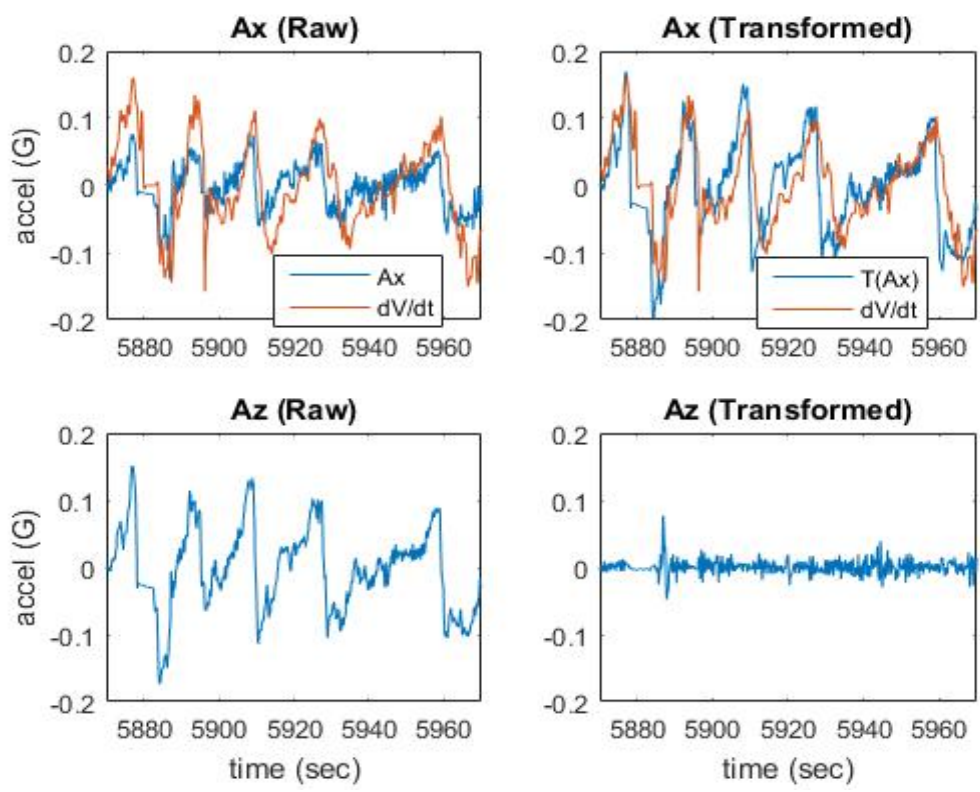

Figure 1. Transformation to normalize acceleration signals

A brute force search was conducted to find the amount of pitch rotation that would minimize the 2-norm of the vertical acceleration signal. An optimal pitch rotation of -61.8 degrees was 
obtained for one participant's complete dataset. The raw and transformed versions of longitudinal and vertical acceleration are shown in Figure 1. While the transformed longitudinal acceleration does not match the speed derivative perfectly, it is a vast improvement over the raw signal. The optimal transformation value must be determined for each vehicle to allow for hardware mounting differences.

\section{Speed Normalization}

We seek to identify driving patterns in naturalistic data that correspond to maneuvers such as turning, slowing, accelerating and parking. However, the time required for all of these maneuvers vary significantly depending on how fast the driver is going. For example, a turn done at $15 \mathrm{mph}$ will obviously take about twice as long as one done at $30 \mathrm{mph}$. Moreover, drivers will sit at zero speed for widely variable times depending on traffic and signal timing. These facts make the identification of consistent patterns quite difficult in the data.

The processing of datasets to put them in a form suitable for time series data mining can sometimes take more time than the activity of data mining itself. A technique called Dynamic Time Warping (DTW) is popular for matching data segments that have similar shapes but are spread out across varying lengths (Rakthanmanon et al., 2013). It allows for data warping to bring two segments into alignment and expose their similarity.

Rather than using Dynamic Time Warping, we have adopted a simple speed normalization to accomplish much the same purpose. We created the distance signal as the integration of speed and then interpolated each signal along a regularly-spaced distance grid, effectively transforming the dependent parameter from time to distance. This process can be thought of as a speed normalization and frames the data much better into a geo-spatial context. Now all right turns look more similar even if the speed varies and even if the driver sits at a light for some indeterminate length of time.

\section{Motif Discovery}

The lateral and longitudinal acceleration were coded into symbols using the SAX method in a sliding window that ran across the dataset one frame at a time. A multidimensional SAX code was achieved simply by concatenating the codes for longitudinal and lateral acceleration together. The speed normalization was performed and a distance grid with one meter spacing was created. A SAX code with six symbols (letters a-f) was used where each letter represented the aggregate mean across five meters. Subsequences of 100 meters were used resulting in SAX words for lateral and longitudinal acceleration that were 20 symbols long each. Each subsequence was normalized by subtracting its mean and dividing by its standard deviation before coding. This allows patterns to be detected even if differences in scale and bias exist. The method of random projects was implemented to locate motifs. Additionally, the matrix profile was used to identify motifs in the data. The method adapted to multidimensional data by adding together the distance metric of each data signal before the final generation of the matrix profile. A 150 meter subsequence length was used. 


\section{RESULTS}

A SAX motif corresponding to a left turn is shown in Figure 2. The raw acceleration signals are shown in the upper right plot while the coded SAX word for the subsequence is given in the figure caption. The longitudinal and lateral acceleration words are concatenated to create a 40 symbol word. The speed profile for this turn is shown in the lower right plot, and the gps position trace is shown in the left plot.
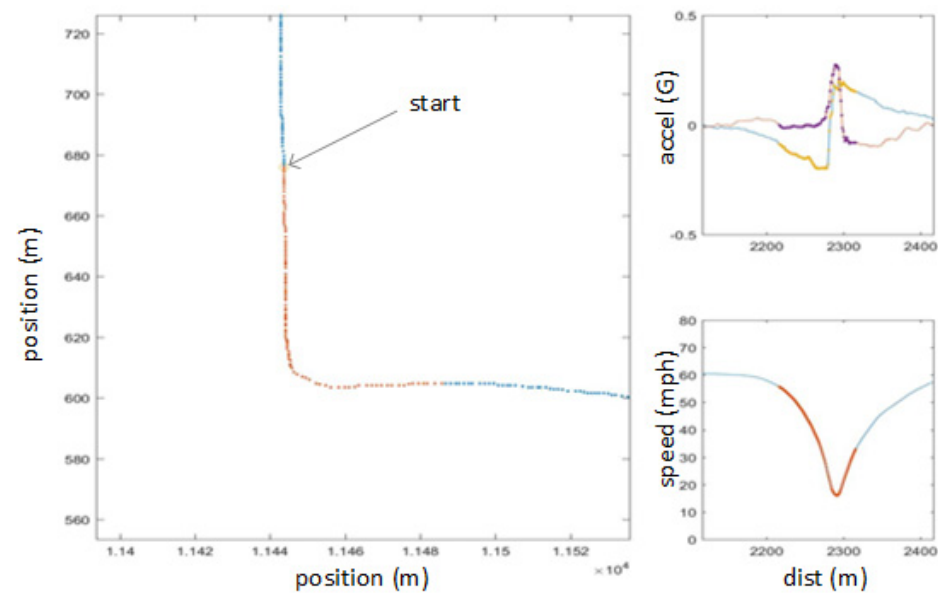

Figure 2. SAX motif with the code: ccbbbbbbbbaabefffffeccceccccedefffcaaa
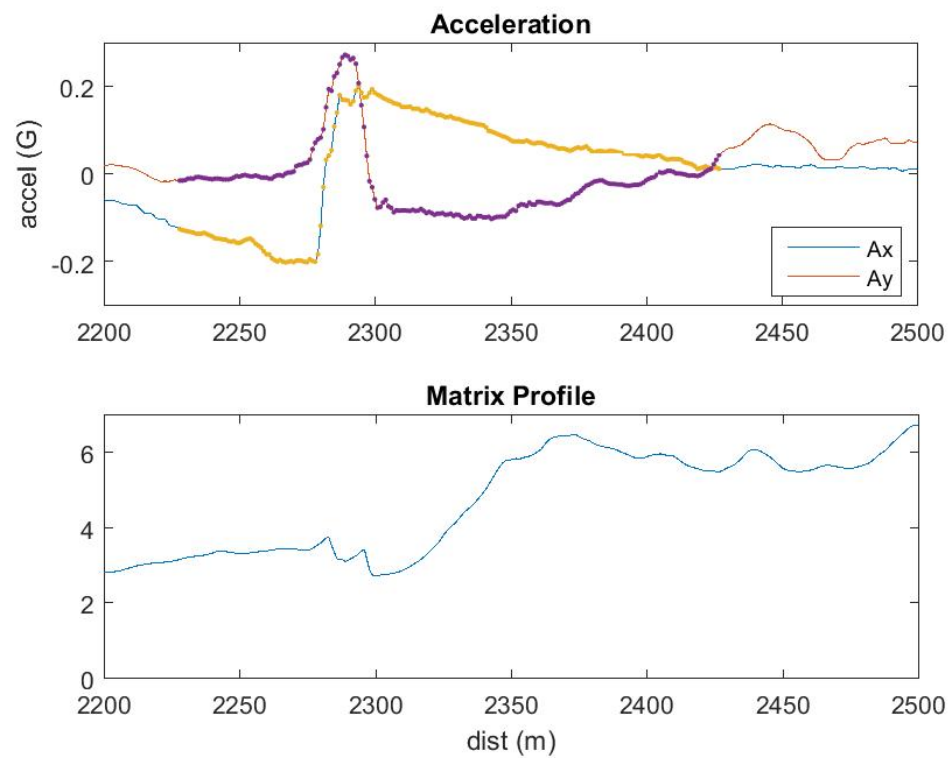

Figure 3. Motif found using the matrix profile

Almost exactly the same motif was obtained using the matrix profile, but with a $150 \mathrm{~m}$ subsequence length instead of a $100 \mathrm{~m}$ length (see Figure 3). One looks for relatively low values of the matrix profile, perhaps even local minima, to suggest good candidates for motifs. The process of searching for motifs can be completely automated, or it can be supervised with the researcher selecting some motifs. 
It was thought that 150 meters might capture more complete maneuvers, and both choices capture several useful motifs. It was observed that instances of the turning motif pictured above included not only these well-defined turns, but also much more mild versions of the pattern with smaller magnitude accelerations at higher speeds. This happens because of the normalization that gets applied to each subsequence. This normalization is necessary in order to capture similar shapes that occur at different scales and offsets in the data. One potential way to eliminate the high speed instances from the motif would be to include speed as an additional dimension to add in the speed profile. Turns have the distinct dip in speed while high speed instances have essentially flat speed. Another way would be to simply use minimum speed to break the motif into two distinct ones.

\section{CONCLUSION}

Two methods of time series data mining, SAX and the matrix profile, were applied to naturalistic driving data. The use of longitudinal and lateral acceleration signals was sufficient to detect many motifs related to driving maneuvers. The detection of such maneuvers can be used to learn typical driving behavior in those maneuvers. Our intent is to then detect when driver behavior in a classified maneuver becomes abnormal.

The SAX method has the advantage of significantly compressing down the size of the data, but at the expense of resolution. Computing the distance between SAX words only gives an accurate lower bound to the Euclidian distance between the subsequences. Implementing the SAX method and random projections for motif discovery requires setting many parameters, including the number of symbols, the number of samples aggregated into each symbol, and several others. The implementation of SAX and random projections in Matlab took a great deal of effort.

The matrix profile method is much more recent than SAX and takes advantage of many advances in time series data mining that speed up the algorithms. It does not sacrifice any data resolution and still runs as fast, or faster, than SAX in finding motifs. Other than selecting the subsequence length, the only other parameter to tune was the threshold on similarity for including a subsequence as an instance of a motif. The implementation of matrix profiles is significantly shorter and simpler than that of SAX and random projections. The matrix profile method also provides a way to compare two different sets of data to look for similarities and differences, something that SAX is not able to do.

\section{ACKNOWLEDGMENTS}

The Understanding the Context of Unintended Acceleration was funded by the Toyota Safety Research and Education Program Settlement. All research was developed independently by the University of Iowa. All content, views and conclusions are expressed by the University of Iowa and have not been sponsored, approved or endorsed by Toyota or the plaintiff's class counsel. The author would like to thank Dan McGehee for providing the naturalistic data used in this research. Also, thanks to Eamonn Keogh for providing Matlab code of the matrix profile method. 


\section{REFERENCES}

Brown, T., Lee, J., Schwarz, C., Fiorentino, D., \& McDonald, A. (2014). Assessing the Feasibility of Vehicle-based Sensors to Detect Drowsy Driving (Final Report No. DOT HS 811 886). Washington, DC: NHTSA.

Chiu, B., Keogh, E., \& Lonardi, S. (2003). Probabilistic Discovery of Time Series Motifs. In Proceedings of the Ninth ACM SIGKDD International Conference on Knowledge Discovery and Data Mining (pp. 493-498). New York, NY, USA: ACM.

Enev, M., Takakuwa, A., Koscher, K., \& Kohno, T. (2016). Automobile Driver Fingerprinting. Proceedings on Privacy Enhancing Technologies, 2016(1), 34-51.

Jovanis, P., Aguero-Valverde, J., Wu, K.-F., \& Shankar, V. (2011). Analysis of Naturalistic Driving Event Data. Transportation Research Record: Journal of the Transportation Research Board, 2236, 49-57.

Lee, J. D., Fiorentino, D., Reyes, M. L., Brown, T. L., Ahmad, O., Fell, J., ... Dufour, R. (2010). Assessing the Feasibility of Vehicle-Based Sensors to Detect Alcohol Impairment.

Lin, J., Keogh, E., Wei, L., \& Lonardi, S. (2007). Experiencing SAX: a novel symbolic representation of time series. Data Mining and Knowledge Discovery, 15(2), 107-144.

Lococo, K. H., Staplin, L., Martell, C. A., \& Sifrit, K. J. (2012). Pedal Application Errors (Final Report No. DOT HS 811 597). Washington, D.C.: NHTSA.

McDonald, A., Lee, J., Aksan, N., Dawson, J., Tippin, J., \& Rizzo, M. (2013). The Language of Driving. Transportation Research Record: Journal of the Transportation Research Board, 2392, 22-30.

McGehee, D., Roe, C., Gunter, L., Johnson, P., Watson, T., Boyle, L. N., ... Repa, B. (2014). Understanding Driver Foot Pedal Behavior (Draft Final Report). University of Iowa.

McLaurin, E., McDonald, A. D., Lee, J. D., Aksan, N., Dawson, J., Tippin, J., \& Rizzo, M. (2014). Variations on a theme Topic modeling of naturalistic driving data. Proceedings of the Human Factors and Ergonomics Society Annual Meeting, 58(1), 2107-2111.

Mueen, A., Hamooni, H., \& Estrada, T. (2014). Time Series Join on Subsequence Correlation. In 2014 IEEE International Conference on Data Mining (pp. 450-459).

Rakthanmanon, T., Campana, B., Mueen, A., Batista, G., Westover, B., Zhu, Q., ... Keogh, E. (2013). Addressing Big Data Time Series: Mining Trillions of Time Series Subsequences Under Dynamic Time Warping. ACM Trans. Knowl. Discov. Data, 7(3), 10:1-10:31.

Schwarz, C., Brown, T., \& Gaspar, J. (2016). The Detection of Visual Distraction using Vehicle and Driver-Based Sensors. In Proceedings of the SAE World Congress. Detroit, MI.

Tran, C., Doshi, A., \& Trivedi, M. M. (2011). Pedal error prediction by driver foot gesture analysis: A vision-based inquiry. In 2011 IEEE Intelligent Vehicles Symposium (IV) (pp. 577-582).

Yu, H., \& Langari, R. (2015). A Detection and Warning System for Unintended Acceleration. In Proceedings of the ASME 2015 Dynamic Systems and Control Conference. Columbus, Ohio. 